\title{
Avaliação da Incidência e de Fatores de Risco para a Colite Isquêmica após Reparo de Aneurisma de Aorta Abdominal
}

\author{
Avaliation of Incidence and Risk Factors for Ischemic Colitis Following \\ Abdominal Aortic Aneurism Repair
}

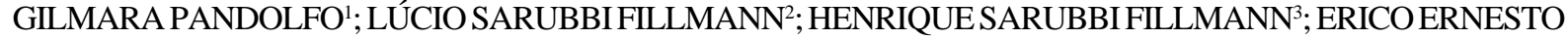 \\ PRETZEL FILLMANN²; JOÃO BATISTAPETRACCO²; MARCOANTÔNIO GOLDANI² \\ ${ }^{1}$ Médica proctologista; ${ }^{2}$ Professores do departamento de cirurgia FAMED-PUCRS; ${ }^{3}$ Médico proctologista do Serviço \\ de colo-proctologia do HSL-PUCRS.
}

PANDOLFO G; FILLMANN LS; FILLMANN HS; FILLMANN EEP; PETRACCO JB; GOLDANI MA. Avaliação da Incidência e de Fatores de Risco para a Colite Isquêmica após Reparo de Aneurisma de Aorta Abdominal. Rev bras Coloproct, 2007;27(3): $288-292$.

\begin{abstract}
RESUMO: Este estudo teve como objetivo principal determinar a incidência da colite isquêmica após o reparo de aneurisma de aorta abdominal, bem como identificar fatores de risco para o desenvolvimento da mesma. Foram estudados 11 pacientes submetidos a reparo cirúrgico eletivo de aneurisma de aorta abdominal no Serviço de Cirurgia Cardiovascular do HSL-PUCRS. A incidência de colite isquêmica foi determinada através de retossigmoidoscopia flexível, com biópsia, realizada em todos os pacientes no $7^{\circ}$ pós-operatório. A incidência da doença foi comparada com variáveis clínicas como: sexo; idade; presença de comorbidades associadas; choque trans-operatório; fluxo na artéria mesentérica inferior (AMI); complicações pós-operatórias; e o desfecho final. Em nossa amostra, a incidência da colite isquêmica após o reparo de aneurisma de aorta abdominal foi 36\%, sendo destes $25 \%$ da forma gangrenosa. A ocorrência de isquemia do cólon foi mais freqüente em associação com o diagnóstico de doença pulmonar obstrutiva crônica, e em pacientes que apresentavam fluxo na artéria mesentérica inferior no pré-operatório $(\mathbf{p}<0,05)$. Em conclusão, neste grupo de pacientes, a incidência de colite isquêmica foi superior à descrita na literatura. A presença de DPOC e pulso na AMI no pré-operatório foram preditivos de ocorrência de colite isquêmica, após o reparo de aneurisma de aorta abdominal.
\end{abstract}

Descritores: isquemia colorretal, aneurisma de aorta, tratamento cirúrgico.

\section{INTRODUÇÃOO}

A colite isquêmica foi inicialmente descrita por Marston, em 1966, como uma entidade clínica caracterizada pela insuficiência de circulação sangüínea no cólon, resultando em vários graus de necrose tecidual local e manifestações sistêmicas (1).

Trata-se de uma infreqüente porém grave complicação da cirurgia de aorta abdominal, sendo o cólon sigmóide o segmento mais afetado $(2,3,4,5)$. Quando é considerado apenas o quadro clínico, a incidência de isquemia colônica após a correção de dilatações aneurismáticas da aorta abdominal é de aproximadamente $1 \%$ a $2 \%(1,2,3,6,7,8,9)$. Quando é realizada retossigmoidoscopia flexível ou colonoscopia pós-operatória de rotina, tais índices chegam a 7\%(10). Hagihara encontrou o desenvolvimento de colite isquêmica em $60 \%$ dos pacientes que realizaram cirurgia para o reparo de aneurisma de aorta abdominal roto (10).

A mortalidade geral dos pacientes com colite isquêmica é de $50 \%$, sendo maior nos casos que necessitam de intervenção cirúrgica (1). Por se tratar de uma entidade clínica infreqüente, de elevada morbimortalidade e com escassos métodos diagnósticos da 
fase aguda, procuramos, através deste estudo, identificar a incidência e a mortalidade da colite isquêmica após a correção de aneurismas de aorta abdominal em nosso meio, e avaliar a existência de possíveis fatores de risco para o desenvolvimento desta complicação.

\section{MATERIAL E MÉTODOS}

Foram estudados 11 pacientes consecutivos que foram submetidos ao reparo cirúrgico eletivo de aneurisma de aorta abdominal no Serviço de Cirurgia Cardiovascular do Hospital São Lucas da PUCRS (HSL-PUCRS) no período de 14 meses do estudo.

Os critérios de exclusão foram a realização da cirurgia em caráter de emergência, e o não fornecimento do consentimento informado pelo paciente.

A avaliação da incidência da colite isquêmica foi determinada através da realização de retossigmoidoscopia flexível, com biópsia, pelo Serviço de Coloproctologia do HSL-PUCRS, em todos os pacientes, no $7^{\circ}$ pós-operatório da cirurgia de reparo de aneurisma de aorta abdominal. Foram considerados achados endoscópicos característicos de isquemia colônica, a presença de sufusões hemorrágicas na submucosa, associadas ou não à formação de úlceras, e a presença de necrose da mucosa com ou sem extensão à parede do intestino

Os achados endoscópicos foram também comparados com as seguintes variáveis do estudo: sexo; idade; presença de comorbidades associadas no préoperatório (diabete mellitus, dislipidemia, hipertensão arterial sistêmica, doença pulmonar obstrutiva crônica, tabagismo, cardiopatia isquêmica, história de infarto agudo do miocárdio, insuficiência renal e acidente vascular encefálico); choque no período trans-operatório; presença/ausência de fluxo na artéria mesentérica inferior (AMI), avaliado através da arteriografia no préoperatório e palpação do vaso no trans-operatório; complicações pós-operatórias; e o desfecho final.

Os resultados foram analisados através da aplicação do Teste exato de Fisher. Valores com $p<0,05$ foram interpretados como indicativos de significância estatística.

\section{RESULTADOS}

Dos 11 casos estudados, apenas um (9\%) era do sexo feminino. A média de idade foi 64 anos. Com relação às comorbidades, a hipertensão arterial sistêmica foi o diagnóstico mais freqüente $(73 \%)$, seguida de cardiopatia isquêmica (55\%), dislipidemia $(46 \%)$, diabete mellitus (30\%), doença pulmonar obstrutiva crônica (27\%), infarto agudo do miocárdio (9\%), insuficiência renal (9\%) e insuficiência cardíaca $(9 \%)$. Além disso, $64 \%$ dos pacientes estudados eram tabagistas.

Com relação à incidência de isquemia colônica, quatro dos 11 indivíduos analisados apresentaram evidência endoscópica e anátomo-patológica de sofrimento vascular do intestino. Um destes pacientes (25\%) apresentou manifestações clínicas de colite isquêmica em sua forma gangrenosa, necessitando de intervenção cirúrgica. Nos três casos restantes (75\%), os achados endoscópicos foram compatíveis com a forma leve e transitória da doença, sendo observadas petéquias e sufusões hemorrágicas na mucosa e submucosa, sendo o diagnóstico corroborado por anátomo-patológico.

A doença pulmonar obstrutiva crônica (DPOC) se mostrou uma condição clínica fortemente relacionada com o desenvolvimento da colite isquêmica. Em nossa série, todos os portadores de DPOC desenvolveram isquemia colônica no pós-operatório. Entre os 8 pacientes restantes sem enfermidades pulmonares crônicas incluídos em nossa casuística, apenas um desenvolveu a colite $(12,5 \%)$. A comparação entre a incidência de isquemia colônica entre os grupos com e sem DPOC se mostrou estatisticamente significativa ( $\mathrm{p}<0,02)$. (figura 1$)$.

Em relação ao fluxo sangüíneo pré-operatório na artéria mesentérica inferior (AMI), dos cinco pacientes (45,5\% da amostra) que apresentavam pulso na AMI previamente à correção do aneurisma, quatro (80\%) desenvolveram isquemia colônica no pós-operatório com a ligadura do vaso. Em comparação, não foram observados casos de isquêmia colônica entre os

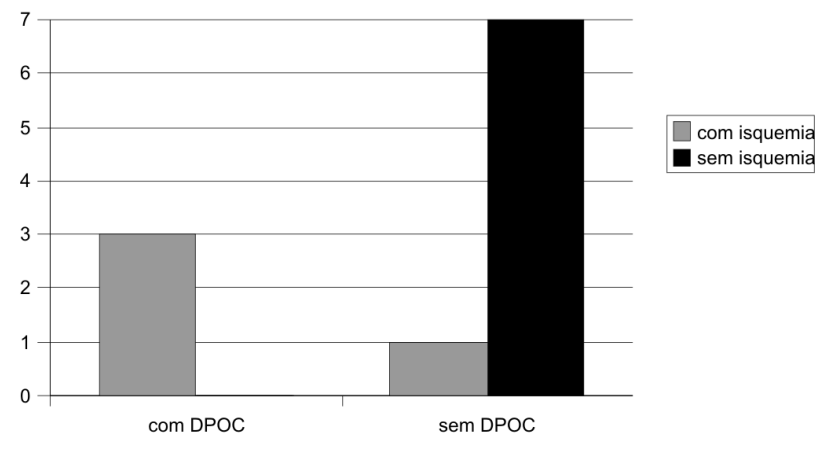

Figura 1 - Ocorrência de isquemia associada à DPOC. 
seis indivíduos cuja AMI se encontrava obstruída desde o período pré-operatório (54,5\% da amostra) $(\mathrm{p}<0,01)$ (figura 2 ).

Todas as demais variáveis do estudo, quando comparadas com a ocorrência ou não de colite isquêmica, não apresentaram resultados estatisticamente significativos.

Dentre os indivíduos estudados, apenas um paciente com isquemia do cólon, no caso em sua forma gangrenosa, evoluiu para óbito.

\section{DISCUSSÃO}

Em geral, os pacientes acometidos pela isquemia colônica são do sexo masculino, idosos, portadores de doença do sistema cardiovascular, diabete mellitus ou insuficiência renal $(1,3,6)$. A apresentação clínica pode ser insidiosa, com dor e distensão abdominal(1). A presença de diarréia sanguinolenta de início recente é considerada o sintoma central $(3,9)$. A forma mais grave da colite, que afeta entre $10 \%$ e $20 \%$ dos pacientes, é caracterizada por necrose gangrenosa transmural do segmento colônico $(1,9)$.

Nos casos de colite isquêmica, após reparo de aneurisma de aorta abdominal, o diagnóstico na fase aguda é difícil. A maioria dos casos é diagnosticada quando há extensa necrose ou sepse, com mortalidade de 54\% (11). Em nossa série, a maioria dos casos não apresentava evidência clínica da doença. No único indivíduo que apresentou sintomas relacionados à insuficiência vascular do cólon, a evolução foi fulminante com gangrena do intestino grosso que, finalmente, levou o paciente a óbito. Não há marcadores químicos ou enzimáticos específicos para este evento. A retossigmoidoscopia flexível ou a colonoscopia são os

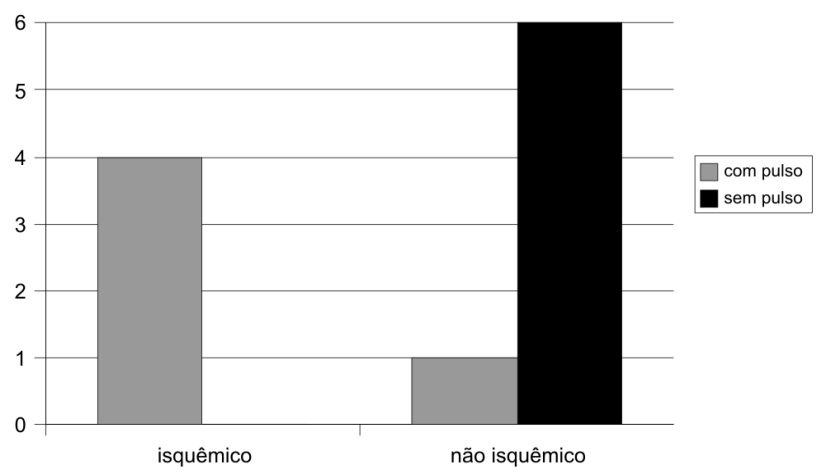

Figura 2 - Isquemia colônica pós ligadura da AMI no trans-operatório. melhores métodos diagnósticos uma vez que também são úteis para graduar a severidade da doença (1). Marston e colaboradores (1) elaboraram uma classificação endoscópica para a colite isquêmica. Segundo os autores, há uma forma isquêmica transitória, em geral aguda, na qual a mucosa é pálida, com edema, congestão e petéquias. A forma intermediária, estenosante, é caracterizada pela fibrose da camada muscular com conseqüente estreitamento do lúmen intestinal. Por fim, temos a forma gangrenosa em que está presente a necrose tecidual extensa (1).

A biópsia, caracterizada pela destruição das criptas, edema e adelgaçamento da mucosa, trombose capilar, hemorragia submucosa, depósitos de hemossiderina, além da presença de células inflamatórias $(1,3)$, pode ser útil no diagnóstico diferencial com outras colites. A arteriografia raramente é diagnóstica. A tomografia computadorizada pode ser normal em pacientes com infarto isquêmico precoce e apresenta alterações em apenas um terço dos pacientes com colite isquêmica estabelecida (1). A ressonância nuclear magnética é pouco utilizada nestes casos e o enema baritado, nos dias de hoje, é pouco solicitado na investigação de abdômen agudo, sendo a "impressão do polegar" na mucosa colônica um sinal radiológico clássico da colite isquêmica neste exame(1). Recentemente, a cintilografia com leucócitos marcados com Iodo111 tem sido descrita como útil para o diagnóstico precoce da colite isquêmica (1). O lavado peritoneal com avaliação laboratorial do líquido coletado pode revelar informações úteis para o diagnóstico. Entretanto, a amilase e o número de leucócitos elevados no líquido do lavado não é um achado específico (1). A laparoscopia pode ser utilizada nos casos duvidosos (1).

$\mathrm{Na}$ cirurgia de reparo de aneurisma de aorta abdominal, a isquemia colônica é uma complicação pouco freqüente, porém com alto potencial de morbimortalidade. Sua incidência é de aproximadamente 1\% a $2 \%$ quando são considerados os sinais clínicos da doença $(1,2,3,6,7,8,9)$. Revisões endoscópicas, mesmo em pacientes assintomáticos, demonstraram uma incidência de 7\% (10). Em nossa amostra, a incidência da colite isquêmica após reparo de aneurisma de aorta abdominal foi superior à descrita na literatura (36\%). Uma vez que não existem marcadores clínicos específicos, um grau elevado de suspeição deve ser observado para que o diagnóstico não ocorra somente quando já existe necrose tecidual. $\mathrm{O}$ desenvolvimento da isquemia após reparo de aneurisma de aorta abdomi- 
Rev bras Coloproct Julho/Setembro, 2007
Avaliação da Incidência e de Fatores de Risco para a Colite Isquêmica após Reparo de Aneurisma de Aorta Abdominal Gilmara Pandolfo e Cols.
Vol. 27 nal, está associado com fatores hemodinâmicos relacionados ao paciente, como a presença de choque no pré-operatório, cirurgia em caráter de emergência e doenças sistêmicas simultâneas $(6,7)$. Evitar o clampeamento aórtico prolongado assim como o sangramento excessivo são medidas importantes na prevenção da colite isquêmica $(6,7)$.

Um fato de importância considerável é o de que a artéria mesentérica inferior (AMI) está permeável em apenas $24 \%$ dos pacientes com aneurisma de aorta abdominal (6). Em vista disso, a AMI freqüientemente é ligada e não reimplantada na correção do aneurisma. Nos casos em que o vaso ainda apresenta fluxo sangüíneo, a falta de tempo para o desenvolvimento de uma circulação colateral eficiente, como a que ocorre na obstrução de evolução mais crônica associada ao desenvolvimento do aneurisma de aorta, pode ser a razão para o surgimento da isquemia. Em nosso estudo, todos os casos de isquemia colônica no pós-operatório apresentavam fluxo através da AMI, na avaliação pré e trans-operatória. Entre aqueles que não apresentaram colite, somente um indivíduo tinha pulso na artéria mesentérica inferior. Logo, é recomen- dada a verificação do fluxo deste vaso através da realização da arteriografia no pré-operatório, ou ecografia no trans-operatório, ou então, pela palpação da artéria durante $o$ ato cirúrgico, como rotina, para doença aneurismal $(1,2,6)$.

Dentre as demais variáveis estudadas, a doença pulmonar obstrutiva crônica (DPOC) se mostrou fortemente associada à isquemia colônica. Não encontramos referências semelhantes na literatura. Vale, entretanto, a recomendação de atenção aos indivíduos portadores de DPOC quanto ao risco de desenvolvimento de colite no período pós-operatório de correção de aneurisma de aorta abdominal.

\section{CONCLUSÕES}

A incidência da colite isquêmica em nosso estudo foi superior à descrita na literatura. A presença de doença pulmonar obstrutiva crônica e de pulso na artéria mesentérica inferior no pré e trans-operatório foram preditivos de ocorrência de colite isquêmica após o reparo de aneurisma de aorta abdominal na amostra estudada.

ABSTRACT: Ischemic colitis is a relatively uncommon complication following abdominal aortic aneurism repair. However, patients with this disease have higher rates of mortality when compared to ischemic colitis alone. The objective of our study is to investigate the incidence and risk factors associated with the occurrence of ischemic colitis following abdominal aortic aneurism repair. We studied eleven patients treated by surgery for aortic aneurism at Hospital São Lucas da PUCRS (HSL-PUCRS). The patients were evaluated for other clinical conditions and were submitted to flexible reto-sigmoidoscopy at the seventh day after surgery. Incidence of ischemic colitis was $36 \%$ and it was associated with chronic obstructive pulmonar disease and the presence of blood flow through the inferior mesenteric artery before surgery. Among the patients with colitis, $25 \%$ presented the gangrenous form of the disease. Mortality was $\mathbf{2 5 \%}$, and $\mathbf{7 5 \%}$ of the patients have only endoscopic manifestations. In conclusion, incidence of ischemic colitis in our study was higher than observed in the literature and it was associated with chronic obstructive pulmonar disease and the presence of blood flow through the inferior mesenteric artery before the surgical treatment for the aneurism.

Key words: colorectal ischemia; aortic aneurism; surgical treatment.

\section{REFERÊNCIAS}

1. Toursarkissian B et al. Ischemic colitis. Surg-Clin-North-Am; 1997, 77(2): 461-70.

2. Bayne $\mathrm{SR}$ et al. A rare complication in elective repair of na abdominal aortic aneurysm: multiple transmural colonic inferts secondary to atheroemboli. Am-Vasc-Surg; 1994, 8(3): 290-5.

3. Longo WE et al. Ischemic colitis complicating abdominal aortic aneurysm surgery in the U.S. veteran. J-Surg-Res; 1996, 60(2): 351-4
4. Sandinson AJP et al. A 4-year prospective audit of the cause of death after infrarenal aortic aneurysm surgery. Br-J-Surg; 1996, 83(10): 1386-9.

5. Valentine RJ et al. Gastrointestinal complications after aortic surgery. J-Vasc-Surg; 1998, 28(3): 404-11.

6. Piotrowski JJ et al. Colonic ischemia: the achilles heel of ruptured aortic aneurysm repair. Am-Surg; 1996, 62(7): 557-60.

7. Bjorck $\mathrm{M}$ et al. Risk factors for intestinal ischaemia after aortoiliac surgery: a combined cohort and case-control study of 2824 operations. Eur-J-Vasc-Endovasc_surg; 1997, 13(6): 531-9. 
8. Mackay $\mathrm{C}$ et al. Case report: rectal infarction after abdominal aortic surgery. Br-J-Radiol; 1994, 67(797): 497-8.

9. Bjorck $\mathrm{M}$ et al. Incidence and clinical presentation of bowel ischaemia after aortoiliac surgery - 2930 operations from a population-based registry in Sweden. Eur-J-Endovasc-Surg; 1996, 12(2): 139-44.

10. Hagihara PF et al. Incidence of ischaemic colitis following abdominal aortic reconstruction. Surg-Gynaecol-Obstet; 1979, 149: 571-3.
11. Poeze $\mathrm{M}$ et al. D-lactate as na early marker of intestinal ischaemic after ruptured abdominal aortic aneurysm repair. Br-J-Surg; 1998, 85(9): 1221-4.

\section{Endereço para correspondência:}

Av. Ipiranga, 6690

Centro Clínico HSL-PUCRS, conjunto 307

Porto Alegre - RS

90610-000

Tel.: 3336-5254 